\title{
SMOOTHLY ATTACHING BOW FLOWS WITH CONSTANT VORTICITY
}

\author{
S. W. MCCUE ${ }^{1}$ and L. K. FORBES ${ }^{2}$
}

(Received 28 January 1998; revised 13 November 1998)

\begin{abstract}
The free surface flow of a finite depth fluid past a semi-infinite body is considered. The fluid is assumed to have constant vorticity throughout and the free surface is assumed to attach smoothly to the front face of the body. Numerical solutions are found using a boundary integral method in the physical plane and it is shown that solutions exist for all supercritical Froude numbers. The related problem of the cusp-like flow due to a submerged sink in a comer is also considered. Vorticity is included in the flow and it is shown that the behaviour of the solutions is qualitatively the same as that found in the problem described above.
\end{abstract}

\section{Introduction}

Two-dimensional bow flows have been given much consideration over the years with the hope of reaching a greater understanding of the nature of flows about wide bodies, such as barges. These bow flows can be divided into two groups, depending on the behaviour of the free surface when it intersects the body. The first is when the free surface meets the body at a stagnation point, while the second is when the free surface attaches to the body smoothly. This paper is concerned with the latter configuration where we consider the flow of a rotational fluid past a semi-infinite body in a fluid of finite depth.

In the stagnant attachment case, irrotational bow flows with no waves upstream have been found by Vanden-Broeck [14] for a finite depth fluid. These results have been extended by McCue and Forbes [12] to allow the fluid to have constant vorticity. All these solutions have been found for supercritical Froude numbers. For subcritical Froude numbers, McCue and Forbes have also found flows with waves. As these

\footnotetext{
${ }^{1}$ School of Mathematical Sciences, University of Nottingham, University Park, Nottingham NG7 2RD, United Kingdom; e-mail: Scott.McCue@nottingham.ac.uk

${ }^{2}$ School of Mathematics and Physics, University of Tasmania, GPO Box 252-37, Hobart, Tas 7001, Australia; e-mail: Larry.Forbes@utas.edu.au

(C) Australian Mathematical Society 2001, Serial-fee code 0334-2700/01
} 
solutions do not satisfy the radiation condition upstream, they must represent some sort of stern flow.

Stagnant attachment flows in infinite depth have been found by various authors, see for example [1, 10, 18, 19]. Only Madurasinghe [10], however, has claimed to have found waveless bow flows which approach a uniform stream. The results by Madurasinghe have been disputed by Farrow and Tuck [1], who show that these solutions did exhibit waves, although admittedly with very small amplitude.

In the case of smoothly attaching flows, Farrow and Tuck [1] did find solutions whose free surface was flat upstream. Similar results were first found by Madurasinghe and Tuck [11]. For a finite depth fluid, Hocking [7] has found waveless solutions for all values of the Froude number $F>1$. Here the front face of the bow was inclined at some arbitrary angle.

The present study continues the work by McCue and Forbes [12] and investigates the flow of a rotational fluid past a semi-infinite two-dimensional body. The free surface is assumed to attach to the front face of the body smoothly so that this work is an extension of that done by Hocking [7], for the case of a vertical face. The problem is solved numerically using a boundary integral method in the physical plane and waveless solutions are found to exist for all supercritical Froude numbers. No solutions with waves could be found.

In the limit as the height of the body above the horizontal bottom approaches zero, the problem is the same as that for the flow due to a line sink in a corner beneath a cusp-like free surface. These flows have been calculated in the past by Hocking [4,5] and Vanden-Broeck and Keller [17] for the case when the flow is irrotational. This problem involving a cusped free surface due to a line sink is also considered here as a special limiting flow, but it is assumed that the fluid has a constant vorticity throughout and that there is uniform shear flow upstream. It is found that the results are qualitatively similar to those obtained for the bow flow case considered earlier in this paper.

\section{Bow flows with smooth attachment}

2.1. Mathematical formulation We consider the steady flow of a rotational fluid past a semi-infinite rectangular body, as shown in Figure 1. It is assumed that the fluid is inviscid and incompressible and that the vorticity $\omega$ is constant throughout the flow. Upstream, the flow approaches a uniform shear flow with speed $c$ on the horizontal bottom and speed $c+\omega H$ on the free surface, which is at the constant height $H$ above the bottom. The corner of the body is rounded, with radius $R$, and the height of the body above the bottom is $D$. Finally, it is assumed that the free surface attaches smoothly to the front face of the body. 


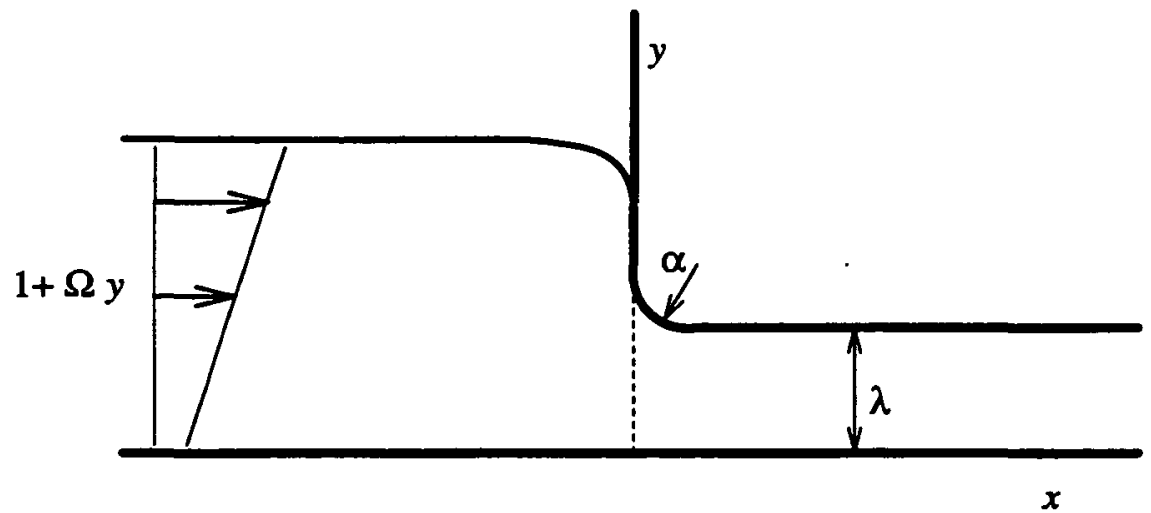

FIGURE 1. A definition sketch in non-dimensional variables of the free surface flow of a rotational fuid past a semi-infinite body.

A Cartesian coordinate system is introduced so that the $x$-axis lies on the horizontal bottom and the $y$-axis overlaps the front face of the body. The problem is now nondimensionalised so that all lengths are scaled with respect to the upstream fluid depth $H$ and all velocities are scaled with respect to the upstream bottom speed $c$. Given this choice of non-dimensionalisation, the problem depends on four parameters. These are the Froude number $F=c / \sqrt{g H}$ which is a measure of the fluid speed upstream when there is no vorticity, the dimensionless vorticity parameter $\Omega=\omega H / c$, the dimensionless gap height $\lambda=D / H$ and the dimensionless circle radius $\alpha=R / H$. Here $g$ is the acceleration due to gravity.

It can be shown from the linear dispersion relation (see McCue and Forbes [12]) that the critical Froude number using these parameters is $F=1 / \sqrt{1+\Omega}$. For Froude numbers less than this critical value, linear theory predicts that periodic, steady waves may exist on the free surface. However, for Froude numbers greater than the critical value, these waves are not possible. When the vorticity $\Omega=0$, the flow is irrotational, and the critical Froude number reduces to $F=1$, which is as expected.

The flow considered here is rotational and hence can be described by the nondimensional stream function $\psi(x, y)$ that satisfies

$$
\nabla^{2} \psi=\Omega
$$

throughout the flow region. The problem is simplified by noting that if we write

$$
\psi(x, y)=y+\frac{1}{2} \Omega y^{2}+\Psi(x, y),
$$

then the function $\Psi$ satisfies Laplace's equation $\nabla^{2} \Psi=0$. This act of subtracting a particular solution of (1) has been used by other authors in the study of surface waves 
with constant vorticity (see for example Teles da Silva and Peregrine [13], VandenBroeck [15] and Forbes and Belward [3]). It follows from (2) that the complex quantity

$$
\chi(z)=U-i V=\Psi_{y}+i \Psi_{x}
$$

is an analytic function of $z=x+i y$ in the flow domain and that the $x$ and $y$ components of the velocity vector can be expressed as

$$
\begin{aligned}
& u(x, y)=\frac{\partial \psi}{\partial y}=1+\Omega y+U(x, y), \\
& v(x, y)=-\frac{\partial \psi}{\partial x}=V(x, y) .
\end{aligned}
$$

Also, there exists a harmonic conjugate function $\Phi(x, y)$ such that both $\Phi$ and $\Psi$ satisfy the Cauchy-Riemann equations, since $\Psi$ is a solution to Laplace's equation.

We solve the problem in the physical plane by using the integral equation method employed by McCue and Forbes [12] in the similar problem of stagnant attachment bow flows. This integral equation method is similar to that used by many other authors, such as Forbes [2] and Vanden-Broeck [15] and is therefore only described briefly here.

The free surface and body are parameterised by means of an arclength $s$. We set $s=0$ at the attachment point so that $s \rightarrow-\infty$ far upstream on the free surface and $s \rightarrow \infty$ far downstream on the body. Each point on the free surface and body can now be written as $(x(s), y(s))$, where the functions $x(s)$ and $y(s)$ are related by the arclength condition

$$
x^{\prime}(s)^{2}+y^{\prime}(s)^{2}=1 .
$$

Cauchy's integral formula can now be applied to the function $\chi$ (defined by (3)) to give

$$
\oint_{\Gamma} \frac{\chi(z(t)) z^{\prime}(t)}{z(t)-z(s)} d t=0
$$

Here the path $\Gamma$ consists of the free surface and body, except for the fixed point $(x(s), y(s))$ which is by-passed by a semicircle of vanishingly small radius, the image free surface and body obtained by reflecting the flow region about the $x$-axis, and two connecting lines at $x= \pm \infty$. After making use of the reflection conditions, the imaginary part of the integral equation (6) can be simplified to give

$$
\begin{aligned}
\pi U(s)= & f_{-\infty}^{\infty} \frac{\Phi^{\prime}(t)(y(t)-y(s))-\Psi^{\prime}(t)(x(t)-x(s))}{(x(t)-x(s))^{2}+(y(t)-y(s))^{2}} d t \\
& +\int_{-\infty}^{\infty} \frac{\Phi^{\prime}(t)(y(t)+y(s))-\Psi^{\prime}(t)(x(t)-x(s))}{(x(t)-x(s))^{2}+(y(t)+y(s))^{2}} d t
\end{aligned}
$$


where $\Phi^{\prime}(t)=U(t) x^{\prime}(t)+V(t) y^{\prime}(t)$ and $\Psi^{\prime}(t)=-V(t) x^{\prime}(t)+U(t) y^{\prime}(t)$. The bar through the first integral indicates that the integral is to be interpreted in the Cauchy Principal Value sense.

This integral equation is to be solved subject to the boundary conditions on the free surface and body. The first of these is the condition of constant pressure on the free surface. This is given by Bernoulli's equation

$$
\frac{1}{2}(1+\Omega y+U(s))^{2}+\frac{1}{2} V(s)^{2}+\frac{1}{F^{2}} y(s)=\frac{1}{2}(1+\Omega)^{2}+\frac{1}{F^{2}},
$$

for $s<0$.

The next condition is the kinematic condition that states that the fluid cannot pass through its own boundary. This implies the stream function $\psi$ is constant on the free surface and body and hence $d \psi / d s=0$ there. It follows from (2) that

$$
\Psi^{\prime}(s)=-(1+\Omega y(s)) y^{\prime}(s),
$$

for all $s$.

Finally, a parametric description of the body is needed. We denote the height of the attachment point by $y_{a}$ so that the arclength at the top of the quarter-circle at the body's nose is $s=s_{b}=y_{a}-(\lambda+\alpha)$ and the arclength at the bottom of the quarter-circle is given by $s=s_{c}=s_{b}+\pi \alpha / 2$. The body can now be described by

$$
x(s)= \begin{cases}0, & 0 \leq s \leq s_{b} \\ \alpha\left[1-\cos \left(\left(s-s_{b}\right) / \alpha\right)\right], & s_{b} \leq s \leq s_{c} \\ s-s_{c}+\alpha, & s \geq s_{c}\end{cases}
$$

and

$$
y(s)= \begin{cases}y_{a}-s, & 0 \leq s \leq s_{b} \\ \lambda+\alpha\left[1-\sin \left(\left(s-s_{b}\right) / \alpha\right)\right], & s_{b} \leq s \leq s_{c} \\ \lambda, & s \geq s_{c} .\end{cases}
$$

The problem is now reduced to solving the integral equation (7) subject to the conditions (5) and (8)-(11) for the unknown functions $x(s), y(s), U(s)$ and $V(s)$. This has to be done numerically and the method for accomplishing this is described in the next section.

2.2. Numerical method The fluid flow is evidently dominated by the behaviour of the variables near the attachment point where $s=0$. It is therefore convenient to make use of an uneven distribution of numerical mesh points, with points clustered 
near $s=0$. A new coordinate $\xi$ is introduced along the free surface and body and points are now spaced evenly in $\xi$, rather than in the original arclength variable $s$. In this section, $\xi$ and $s$ are chosen to be related by the transformation

$$
\xi= \begin{cases}-\ln (1-s), & s<0 \\ \ln (1+s), & s \geq 0\end{cases}
$$

A mesh of $N$ evenly-spaced grid points $\xi_{1}, \xi_{2}, \ldots, \xi_{n a}, \ldots, \xi_{N}$ is placed over the new variable $\xi$, which encompasses both the free surface and the body. Here $\xi_{1}$ and $\xi_{N}$ are chosen to represent $-\infty$ and $\infty$ respectively. The attachment point is fixed at $\xi_{n a}=0$ and we choose $n a=(N+1) / 2$. The grid points are equally spaced in the $\xi$ variable so that they are physically closer together near the attachment point and further apart as $x \rightarrow \pm \infty$.

An initial guess is made for the vector of $(3 N+1) / 2$ unknowns

$$
\mathbf{u}=\left[y_{1}^{\prime}, \ldots, y_{n a}^{\prime} ; \Phi_{1}^{\prime}, \ldots, \Phi_{N}^{\prime}\right]^{T}
$$

All other flow quantities can now be computed. That is, the $x_{i}^{\prime}$ can be found from the arclength condition (5) which, in the new variable $\xi$, takes the form

$$
x^{\prime}(\xi)^{2}+y^{\prime}(\xi)^{2}=e^{2|\xi|},
$$

and the $\Psi_{i}^{\prime}$ can be found from the kinematic condition (9). The coordinate $x$ is found by integrating backwards using the trapezoidal rule and the fact that $x_{n a}=0$ at the attachment point. The coordinate $y$ is obtained by integrating forwards from the first point upstream where $y_{1}=1$, again using the trapezoidal rule. The $x_{i}, y_{i}, x_{i}^{\prime}$ and $y_{i}^{\prime}$ on the body are found from (10)-(11). Finally, the velocities $U_{i}$ and $V_{i}$ can be found from

$$
\begin{aligned}
& U(\xi)=e^{-2|\xi|}\left[x^{\prime}(\xi) \Phi^{\prime}(\xi)+y^{\prime}(\xi) \Psi^{\prime}(\xi)\right], \\
& V(\xi)=e^{-2|\xi|}\left[y^{\prime}(\xi) \Phi^{\prime}(\xi)-x^{\prime}(\xi) \Psi^{\prime}(\xi)\right] .
\end{aligned}
$$

This initial guess for $\mathbf{u}$ is updated iteratively using Newton's method by forcing an error vector $\mathbf{E}(\mathbf{u})$ to zero. The first na components of this error vector are obtained by evaluating the Bernoulli equation ( 8 ) at the points $\xi_{1}, \xi_{2}, \ldots, \xi_{n a}$.

The next $N-1$ components of $\mathbf{E}$ come from evaluating the integral equation (7) at the $N-1$ half-mesh points $\xi_{j+1 / 2}, j=1, \ldots, N-1$. The integrals in (7) are evaluated using trapezoidal rule integration and the singularity can be ignored since the points $\xi_{j+1 / 2}$ have been taken to lie exactly half-way between consecutive mesh points.

It is important to estimate the portions of the integrals in (7) which lie outside our numerical domain of integration. As $\xi \rightarrow-\infty$, the velocities $U$ and $V$ in (4) vanish, 
so there is no contribution for $\xi<\xi_{1}$. However, as $\xi \rightarrow \infty$, the velocity $U$ has some finite value, $\bar{U}=\left[(1+\Omega / 2)-\left(\lambda+\Omega \lambda^{2} / 2\right)\right] / \lambda$, which is determined by considering conservation of mass. We therefore approximate $U$ in (7) by $\bar{U}$ for large $\xi$, and find that the contribution to the integrals in (7) from the region $\xi>\xi_{N}$ is

$$
\bar{U} \arctan \left(\frac{x_{N}-x(\xi)}{|\lambda-y(\xi)|}\right)-\bar{U} \arctan \left(\frac{x_{N}-x(\xi)}{\lambda+y(\xi)}\right) .
$$

Finally, the last component of the error vector enforces the condition of smooth attachment. That is, $y_{n a}^{\prime}=-1$.

A variation of this numerical method is used when the Froude number $F=\infty$. Here the velocity on the free surface is constant and Bernoulli's equation (8) is simply

$$
\frac{1}{2}(1+\Omega y+U(\xi))^{2}+\frac{1}{2} V(\xi)^{2}=\frac{1}{2}(1+\Omega)^{2},
$$

for $\xi<0$.

2.3. Results The numerical method described in the last section was used to calculate many solutions for various values of the parameters $F$ and $\Omega$, for $\lambda=0.15$ and $\alpha=0.15$. We chose to fix the gap height $\lambda=0.15$ and circle radius $\alpha=0.15$, as it was found that varying these parameters did not change the qualitative behaviour of the solutions. In most cases it was found that if we used $N=501$ numerical grid points and chose $s_{1}=-10$ and $s_{N}=10$, then the results were independent of the grid within graphical accuracy. This was not the case only in parameter regions where the numerical scheme was near failure. We therefore use this grid spacing and number of points in all the results presented in this section.

Typical free surface profiles for $\Omega=0$ are shown in Figure 2. Here, four different solutions are shown, for the four Froude numbers $F=1,1.25,2$ and $\infty$ (bottom to top profiles, respectively). As $x \rightarrow-\infty$, the surfaces become flat at $y=1$. It can be seen from this figure that for smaller Froude numbers, the free surface rises from the attachment point and approaches this asymptotic level more slowly than for larger Froude numbers. We also see that the surface profile for $F=2$ is very similar to the profile for $F=\infty$. In fact, the free surfaces for Froude numbers greater than about $F=5$ were found to be almost identical to those for an infinite Froude number. This behaviour was discussed by Hocking [7] in the similar problem where the front face of the body was allowed to be inclined at an arbitrary angle.

In the case where the flow is irrotational $(\Omega=0)$, it was found that solutions with reliable accuracy could be found for all Froude numbers $F>1$. Using the solutions for $F=1$ as a starting guess, a small range of numerical solutions for Froude numbers $F<1$ could also be found, however these solutions were disregarded as they were numerically unreliable. The iteration scheme converged very slowly in this region 


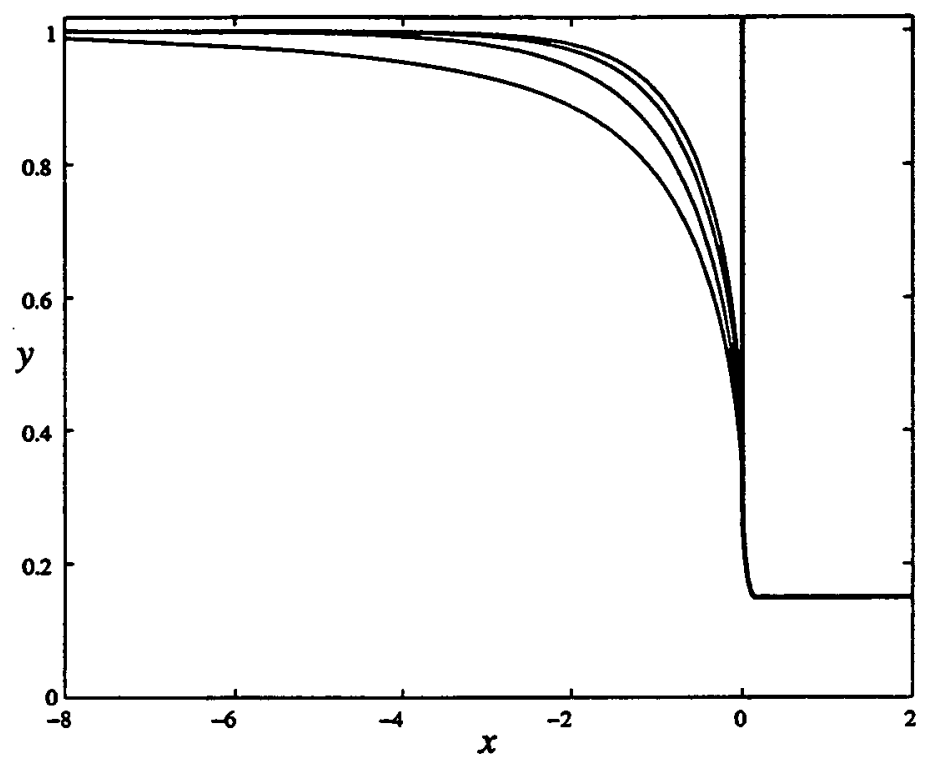

FIGURE 2. Typical free surface profiles for $\Omega=0, \lambda=0.15$ and $\alpha=0.15$. From bottom to top, the surfaces represent solutions found for Froude numbers $F=1,1.25,2$ and $\infty$.

and the resulting free surface profiles were dependent on the grid size and number of numerical grid points. Furthermore, these solutions were characterised by a free surface which appeared to approach asymptotically a level well below $y=1$ in the far field. Thus we conclude that, for irrotational flow, these solutions only exist for $F>1$.

The behaviour of the solutions for any fixed vorticity $\Omega>-1$ was found to be qualitatively the same as that found for $\Omega=0$. Reliable solutions could be found for all Froude numbers greater than some limiting value, $F=F_{L}$ say. Again, as before with the irrotational case, the free surface profiles corresponding to lower Froude numbers approached the asymptotic level $y=1$ more slowly than those for higher Froude numbers. We conclude later in this section that the limiting value of the Froude number $F_{L}$ is in fact the critical Froude number $F=1 / \sqrt{1+\Omega}$.

An interesting quantity found as part of the solution process is the height at which the free surface attaches to the front face of the body, denoted by $y_{a}$. Figure 3 shows the relationship between the attachment height $y_{a}$ and the Froude number $F$ for different values of the vorticity $\Omega$, given that $\lambda=0.15$ and $\alpha=0.15$. It can be seen from this figure that for any fixed $\Omega$, the attachment height increases monotonically with the Froude number. In fact, the height of any point on the free surface was found to increase with the Froude number in this way, given that the vorticity was fixed at some value. 


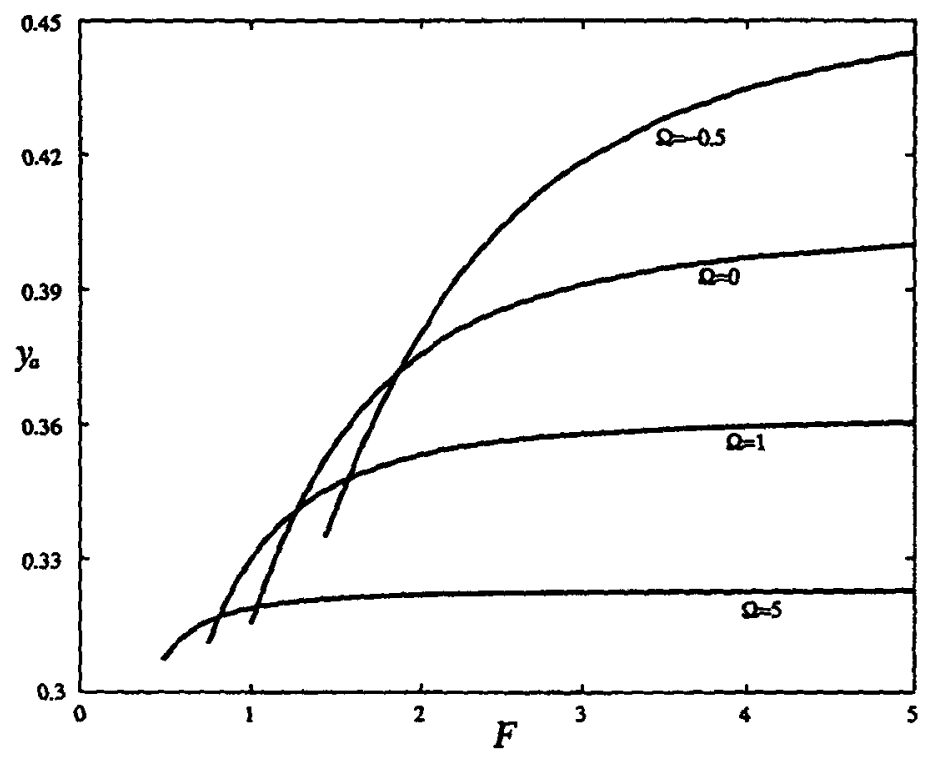

FigURE 3. The dependence of the attachment height $y_{a}$ on the Froude number $F$ for four different vorticity values $\Omega=-0.5,0,1$, and 5 . All solutions are found for $\lambda=0.15$ and $\alpha=0.15$.

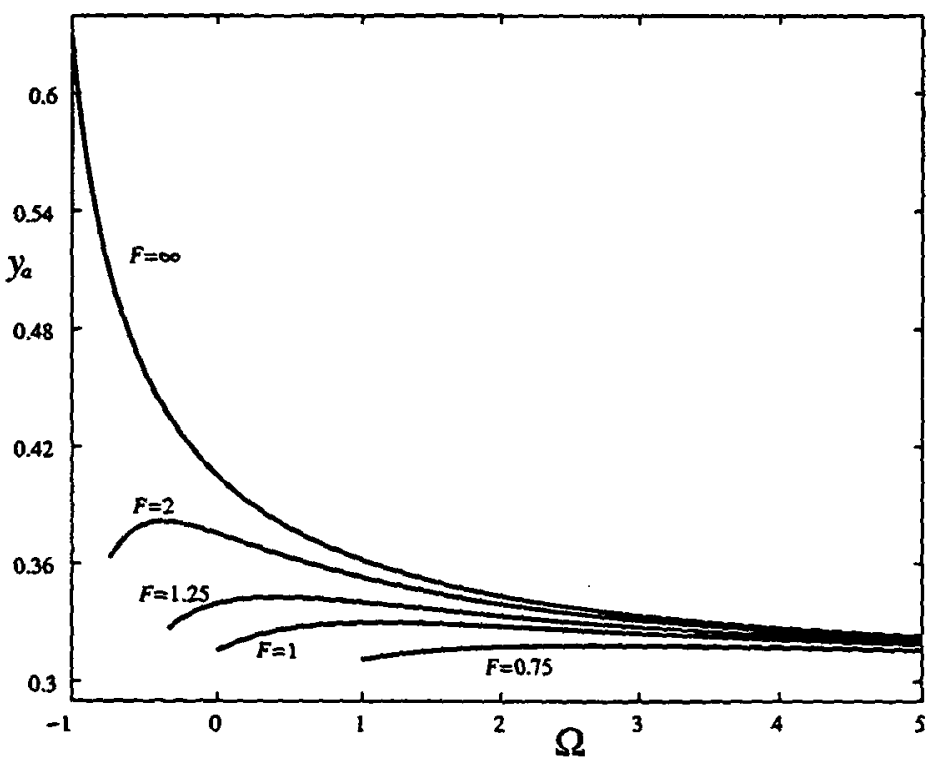

FIGURE 4. The dependence of the attachment height $y_{a}$ on the vorticity $\Omega$ for five different Froude numbers $F=0.75,1,1.25,2$ and $\infty$. All solutions are found for $\lambda=0.15$ and $\alpha=0.15$. 
We now consider the effect that varying the vorticity has on the flow when the Froude number is fixed. Five plots of the attachment height $y_{a}$ versus the vorticity $\Omega$ for different Froude numbers $F$ are shown in Figure 4. In each case, except for $F=\infty$, the attachment height rises to a maximum and then monotonically decreases as $\Omega$ increases. For example, when the Froude number is fixed at $F=2$, the solution with the lowest vorticity is when $\Omega=-0.74$. Here the free surface attaches to the body at $y_{a}=0.363$. After reaching a maximum of $y_{a}=0.382$ at $\Omega=-0.38$, the attachment height decreases as the vorticity $\Omega$ increases.

Three free surface profiles for $F=2$ are shown in Figure 5 for the three values of vorticity $\Omega=0,-0.74$ and 5 . The top profile is the solution for $\Omega=0$ (the irrotational case), the middle profile corresponds to $\Omega=-0.74$ and the bottom one represents the positive vorticity value $\Omega=5$. Note that while the free surface for $\Omega=5$ is lower than that for $\Omega=-0.74$ near the body, it rises to the asymptotic level of $y=1$ faster than the profile for $\Omega=-0.74$.

As can be seen from Figures 3 and 4, the height at which the free surface attaches to the body has little dependence on the Froude number when the vorticity parameter is large. This is to be expected, since at the upstream fluid depth $y=1$, the fluid speed is $u=1+\Omega$, and so when $\Omega \rightarrow \infty$, the shear velocity caused by vorticity is the dominant effect.

Another feature of Figure 4 is the plot for $F=\infty$. Here solutions exist for all vorticity values down to and including $\Omega=-1$. At this value, Bernoulli's equation (12) implies that the whole free surface becomes stagnant. This situation is physically unlikely to happen, although it is nevertheless very interesting.

We end this section by showing in Figure 6 the parameter space where accurate solutions have been computed for the gap height $\lambda=0.15$ and circle radius $\alpha=0.15$. These solutions could be found for all values of $F$ and $\Omega$ in the shaded area, above the solid line. Included in this figure is a dashed curve, which shows a plot of the critical Froude number (found from linear theory) versus vorticity. It can be seen that the solid curve, representing the computed limiting Froude number, matches this dashed curve very closely, and hence we conclude that in fact this limiting Froude number is the critical Froude number. Furthermore, since this parameter space plot is essentially the same for all values of the gap height $\lambda$ and circle radius $\alpha$, we conclude that solutions for this problem exist for all supercritical Froude numbers $F>1 / \sqrt{1+\Omega}$, regardless of the values of $\lambda$ and $\alpha$.

\section{Cusp-like flows due to a sink in a corner}

3.1. Mathematical formulation In this section we consider the special case of the previous problem where both $\lambda \rightarrow 0$ and $\alpha \rightarrow 0$. The resulting flow is that due to a 


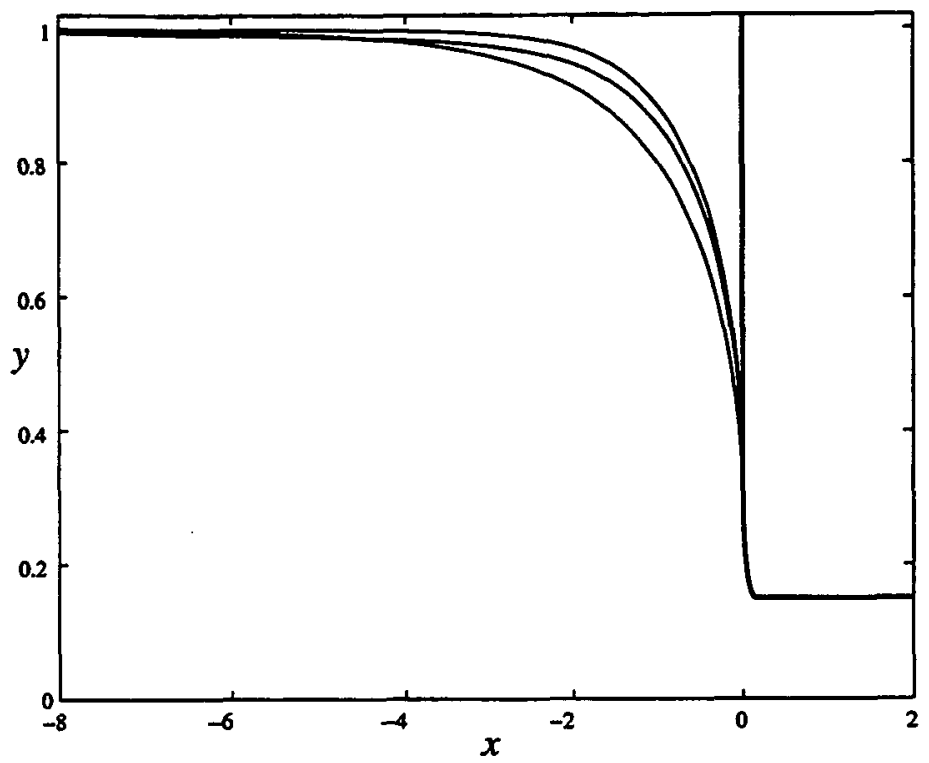

FIGURE 5. Three free surface profiles for $F=2, \lambda=0.15$ and $\alpha=0.15$. From top to bottom near the body, the surfaces represent solutions found for vorticity values $\Omega=0,-0.74$ and 5 .

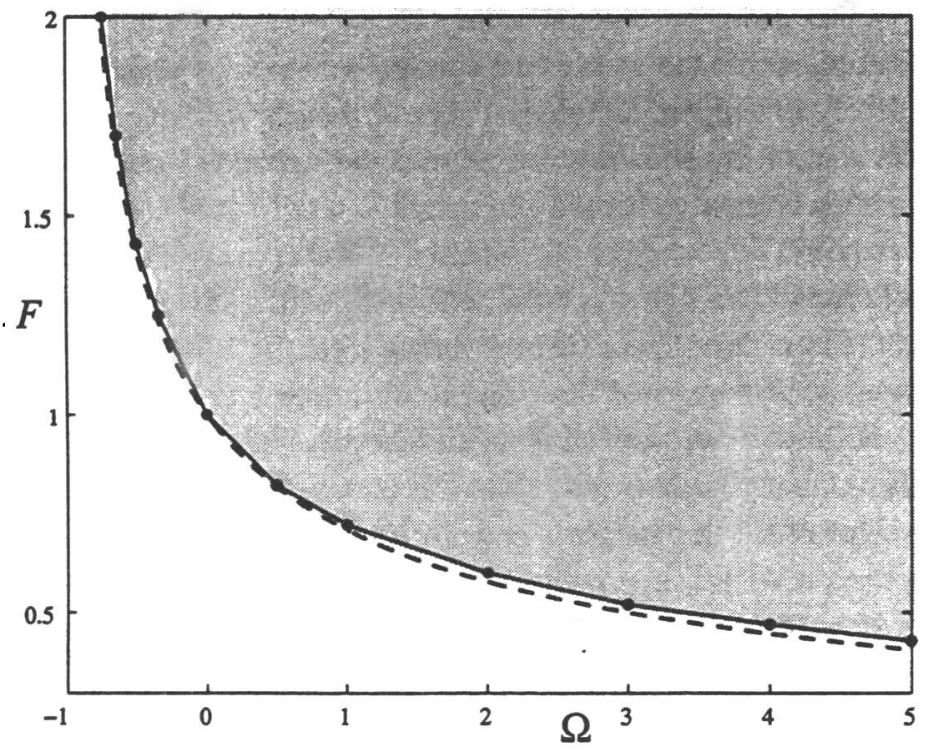

FIGURE 6. A sketch of the parameter space in which accurate solutions have been computed is shown here in the shaded area, for the case where the gap height $\lambda=0.15$ and the circle radius $\alpha=0.15$. The dashed curve represents a plot of the critical Froude number $F=1 / \sqrt{1+\Omega}$ versus the vorticity $\Omega$. 


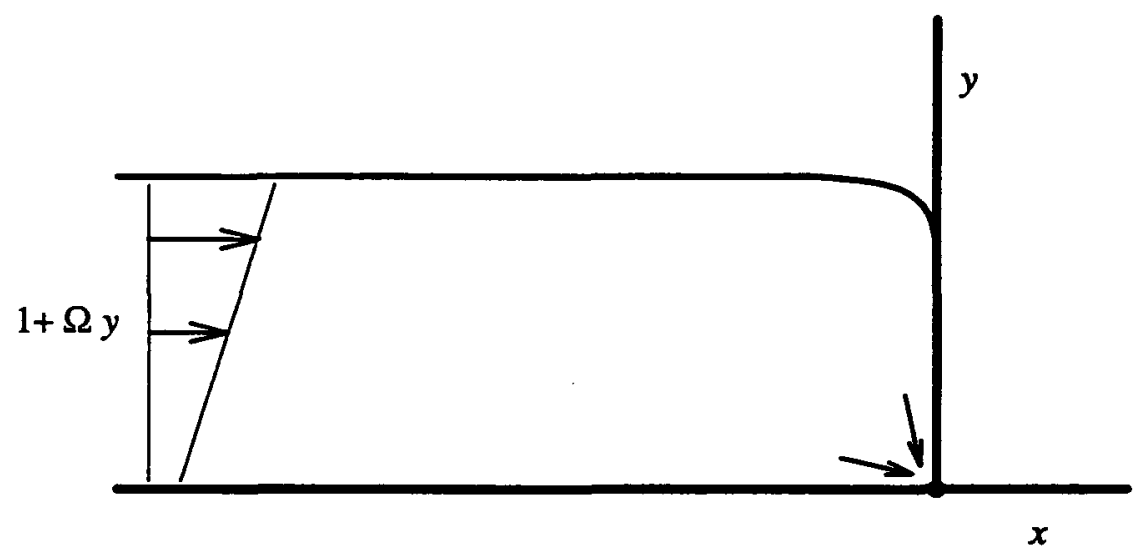

FIGURE 7. A definition sketch in non-dimensional variables of the free surface flow of a rotational fluid due to a submerged sink situated in a 90 degree corner.

line sink situated in a corner, as shown in Figure 7. These flows are often referred to as cusp-like flows, since the free surface forms a cusp directly above the withdrawal point. It should be noted, however, that the free surface cannot enter the sink directly, as the air above the free surface is assumed to be stagnant. The fluid is again rotational with constant vorticity $\Omega$. Upstream the fluid approaches a uniform shear flow in the same way as before, when flow past a semi-infinite body was considered.

A conservation of mass argument is used to show that the strength of the sink must be $1+\Omega / 2$ and hence by (4)

$$
U-i V \sim \frac{-2(1+\Omega / 2)}{\pi} \frac{1}{z}-1 \text { as } z \rightarrow 0 .
$$

As we will later integrate through the sink, the $\chi$ function needs to be redefined so that it is no longer singular there. We therefore let

$$
\chi(z)=U-i V+\left(1+\frac{1}{2} \Omega\right)\left(1+\operatorname{coth} \frac{\pi z}{2}\right)
$$

and note that $\chi(0)=\Omega / 2$. The function $\chi(z)$ is analytic everywhere in the flow domain and vanishes as $x \rightarrow-\infty$.

The free surface and wall together are parameterised with respect to arclength in a similar way to that done in Section 2.1. Again we set $s=0$ at the attachment point (the cusp) so that $s=y_{a}$ at the sink.

Cauchy's integral formula is applied to the new $\chi$ function in (14) to give the relation (6) as before, where this time the contour $\Gamma$ consists of the free surface and vertical wall, except for the fixed point $(x(s), y(s))$ which is by-passed by a semicircle of small radius, the image free surface and wall obtained by reflecting the flow region 
about the horizontal bottom, and a vertical line at $x=-\infty$ connecting the free surface and its image. The imaginary part of the resulting integral equation is, after simplification,

$$
\begin{aligned}
\pi a(s)= & \int_{-\infty}^{y_{a}} \frac{\left[a(t) x^{\prime}(t)-b(t) y^{\prime}(t)\right](y(t)-y(s))}{(x(t)-x(s))^{2}+(y(t)-y(s))^{2}} d t \\
& -f_{-\infty}^{y_{a}} \frac{\left[b(t) x^{\prime}(t)+a(t) y^{\prime}(t)\right](x(t)-x(s))}{(x(t)-x(s))^{2}+(y(t)-y(s))^{2}} d t \\
& +\int_{-\infty}^{y_{a}} \frac{\left[a(t) x^{\prime}(t)-b(t) y^{\prime}(t)\right](y(t)+y(s))}{(x(t)-x(s))^{2}+(y(t)+y(s))^{2}} d t \\
& -\int_{-\infty}^{y_{a}} \frac{\left[b(t) x^{\prime}(t)+a(t) y^{\prime}(t)\right](x(t)-x(s))}{(x(t)-x(s))^{2}+(y(t)+y(s))^{2}} d t
\end{aligned}
$$

where the $a(s)$ and $b(s)$ are the real and imaginary parts of the function $\chi(z(s))$. Here, these functions are given as

$$
a=U+\left(1+\frac{1}{2} \Omega\right)\left(1+\frac{G}{K}\right) \text { and } b=-V-\left(1+\frac{1}{2} \Omega\right) \frac{H}{K},
$$

where

$$
G=\sinh \frac{\pi x}{2} \cosh \frac{\pi x}{2}, \quad H=\sin \frac{\pi y}{2} \cos \frac{\pi y}{2} \quad \text { and } \quad K=\sin ^{2} \frac{\pi y}{2}+\sinh ^{2} \frac{\pi x}{2} .
$$

As usual, Bernoulli's equation (8) holds on the free surface and the kinematic condition (9) holds on the free surface and the vertical wall.

3.2. Numerical method and results As with the numerical method for the bow flows, it is desirable to place a mesh over the free surface such that there are more points near the cusp and fewer points far upstream. This is done by first making the transformation

$$
\xi= \begin{cases}-\ln (1-s), & s<0, \\ s, & s \geq 0 .\end{cases}
$$

We now place a mesh of $M$ grid points $\xi_{1}, \xi_{2}, \ldots, \xi_{M}$ over the free surface and a separate mesh of $N-M$ grid points $\xi_{M}, \ldots, \xi_{N}$ over the vertical wall. Each interval is equally spaced in the $\xi$ variable.

An initial guess is made for the new vector of $N+M-1$ unknowns

$$
\mathbf{u}=\left[y_{1}^{\prime}, \ldots, y_{M}^{\prime} ; \Phi_{1}^{\prime}, \ldots, \Phi_{N-1}^{\prime}\right]^{T},
$$

and as before, given this initial guess, all other flow quantities can be computed using the methods described in Section 2.2. Newton's method is used to solve for 
$\mathbf{u}$ by forcing an error vector $\mathbf{E}(\mathbf{u})$ to zero. The first $N-2$ components of this error vector can be found by evaluating the integral equation (15) at the half-mesh points $\xi_{j+1 / 2}, j=1, \ldots, N-2$. The integrals are evaluated in the same manner as in Section 2.2, however now there are no correction terms that need to be considered. The next $M$ components come from evaluating Bernoulli's equation (8) at the points $\xi_{1}, \xi_{2}, \ldots, \xi_{M}$. Finally, the last component of $\mathbf{E}(\mathbf{u})$ comes from enforcing the cusp condition $y_{M}^{\prime}=-1$.

As before with the bow flows, it was sufficient to use $M=250$ numerical mesh points on the free surface for the results to be independent of the grid within graphical accuracy, given that $s_{1}=-10$. The number of points used on the vertical wall was chosen so that the step size $y_{a} /(N-M)$ was approximately equal to the step size $\xi_{j+1}-\xi_{j}, j=1, \ldots, M-1$, on the free surface.

For fixed vorticity values, solutions exist for all Froude numbers greater than some lower limit $F_{L}$, in the same manner as those found in the earlier bow flow problem. Again, it was found that this lower limit was the critical Froude number $F=1 / \sqrt{1+\Omega}$, which can be determined from linear theory. Also, as with the bow flow problem, the height of each point on the free surface was found to increase monotonically as the Froude number increases.

The behaviour of the solutions when the Froude number is fixed at some value is, however, slightly different to that found in the bow flow problem, although in general the qualitative behaviour is the same. Solutions could be found for vorticity values up to some limiting value, above which the present numerical scheme failed to converge. This upper limit on the vorticity was found to be about $\Omega=2.35$, regardless of the Froude number. It is not clear why this numerical method fails here, since an alternate scheme was able to find solutions for arbitrarily large vorticity values. This alternate numerical scheme was identical to the present one, except that the mesh of grid points on the free surface was evenly-spaced in the physical variable $s$, instead of evenly-spaced in the transformed variable $\xi$.

Another difference in behaviour is in the existence of solutions at infinite Froude number. In the present problem, numerical solutions could not be found for vorticity values near $\Omega=-1$. This is in contrast to the bow flow problem, where solutions could be found for all vorticity values down to $\Omega=-1$ for all gap heights $\lambda$.

Three free surface profiles are shown in Figure 8 for Froude number $F=2$ and vorticity values $\Omega=-0.75,0$ and 2.35. The top profile is the solution for $\Omega=0$, the middle profile near the wall represents the solution for $\Omega=-0.75$ and the bottom one corresponds to $\Omega=2.35$. This figure exhibits the same behaviour as that shown in Figure 5 for the bow flow case where the gap height $\lambda$ and circle radius $\alpha$ at the nose of the bow are nonzero. That is, the free surface corresponding to the positive vorticity is lower than the profile for the negative vorticity near the body, but rises to the asymptotic level of $y=1$ faster than the profile for the negative vorticity. A 


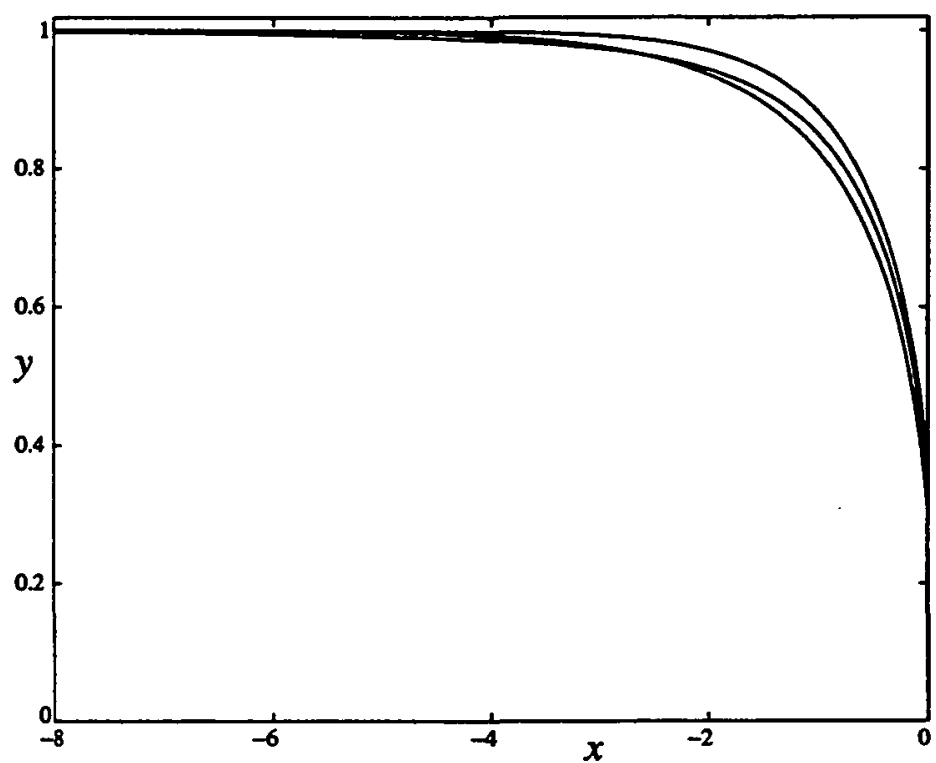

FIGURE 8. Three profiles for $F=2$ when there is a line sink located at the origin beneath a cusped free surface. From top to bottom near the body, the surfaces represent solutions found for vorticity values $\Omega=0,-0.75$ and 2.35 .

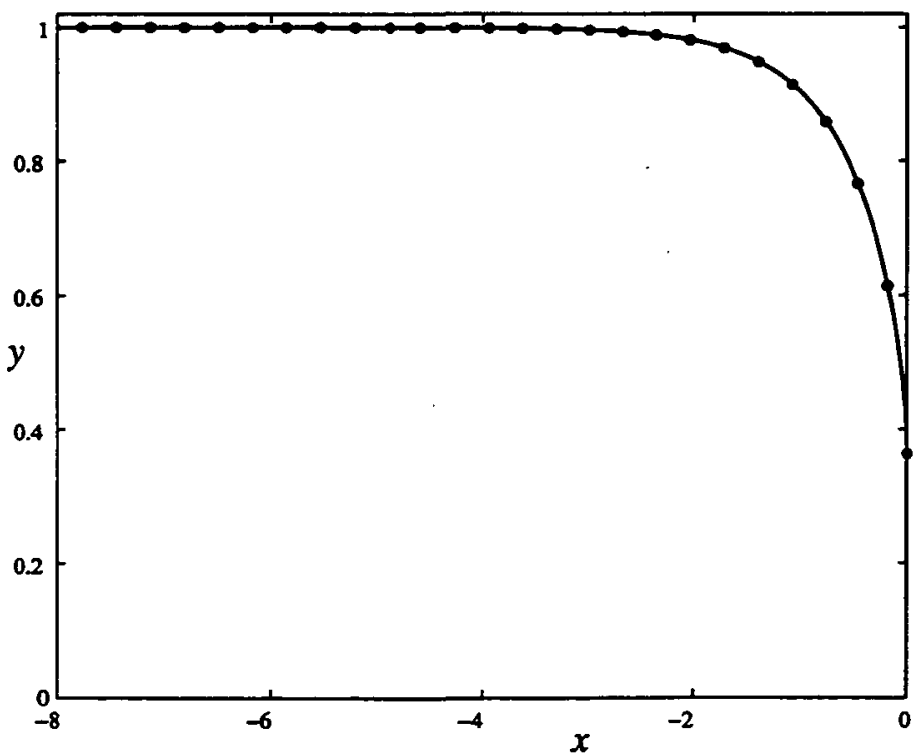

FIGURE 9. A comparison of a numerically found solution and an exact one. The solid free surface represents the solution found using the numerical method in Section 3.2 when $F=\infty$ and $\Omega=0$. The dotted free surface represents an exact solution found by King and Bloor [9]. 
variety of other solutions have been computed for many different values of $F$ and $\Omega$ and, as stated previously, the behaviour of the profiles is qualitatively similar to the behaviour found when studying the bow flow problem in Section 2 .

Finally, to check the accuracy of our numerical method, we compare a solution found with an exact solution derived by King and Bloor [9]. These authors used a generalised Schwarz-Christoffel mapping to show that when $F=\infty$ and $\Omega=0$, the free surface is given exactly by

$$
z=i+\frac{2}{\pi}\left\{\left(\frac{\zeta}{\zeta+1}\right)^{1 / 2}-\arcsin \sqrt{\zeta}-\frac{i}{(\zeta+1)^{1 / 2}}\right\},
$$

where $\zeta$ is the real coordinate of a conformally mapped plane. The free surface can be obtained parametrically from this expression in the form $x(\zeta), y(\zeta)$ for $\zeta>0$. This exact solution and the corresponding one found using our numerical method are shown in Figure 9. We see that both profiles are in excellent agreement with each other, as expected; this agreement represents strong confirmation of the reliability of our numerical scheme.

\section{Conclusions}

In this paper, the free surface flow of a rotational fluid past a semi-infinite body is solved numerically using a boundary integral method. Solutions are found where the surface meets the front face of the body smoothly. These sorts of flows have been calculated before by Hocking [7] for the special situation where the vorticity $\Omega=0$. It is found that for any fixed vorticity value $\Omega>-1$, the behaviour of the solutions is qualitatively the same as that found by Hocking in the irrotational case. That is, solutions can be found for all Froude numbers above and including the critical value $F=1 / \sqrt{1+\Omega}$. When $\Omega=-1$, the only solution is the limiting case, where $F=\infty$.

The non-existence of solutions below this lower Froude number limit is consistent with other withdrawal flows with similar geometry. In most cases when the flow is irrotational $(\Omega=0)$, this lower limit is $F=1$. For example, when studying the problem of fluid flowing into a vertical slot, Hocking [6] found a lower bound of $F=1$ for sufficiently small slot widths. Here the free surface was assumed to attach smoothly to the vertical wall above the slot. Similar behaviour is found with other withdrawal flows where a cusp lies on the free surface directly above a source (see for example $[4,5,17])$.

The question arises as to why no steady solutions could be found below this lower Froude number limit. Possibly the answer lies in the formation of waves on the free surface. In the related problem of the irrotational flow due to a submerged source in a fluid of finite depth, waves have recently been found by Vanden-Broeck [16] 
and Hocking and Vanden-Broeck [8], for $F<1$, by placing a rigid lid on the free surface downstream. They could also reproduce a special family of waveless solutions found by Vanden-Broeck and Keller [17], where the Froude number is found as part of the solution process. If waves do exist in the present problem, then it would be natural to assume they exist in the same way, and that the present numerical method should at least detect a subcritical waveless branch of solutions at a unique Froude number $F<1 / \sqrt{1+\Omega}$, for a fixed vorticity $\Omega$. Unfortunately, as was the case with Hocking [7], no such subcritical family of solutions could be found and the question of whether waves exist for this flow geometry remains unanswered.

\section{Acknowledgements}

The work of the first author has been supported by a University of Queensland Postgraduate Research Scholarship. The authors are indebted to an anonymous referee, whose comments have led to a significant improvement in the final draft of this paper.

\section{References}

[1] D. E. Farrow and E. O. Tuck, "Further studies of stern wavemaking", J. Austral. Math. Soc. Ser. B 36 (1995) 424-437.

[2] L. K. Forbes, "On the effects of non-linearity in free-surface flow about a submerged point vortex", J. Engng Maths 19 (1985) 139-155.

[3] L. K. Forbes and S. R. Belward, "Atmospheric solitary waves: some applications to the moming glory of the Gulf of Carpentaria", J. Fluid Mech. 321 (1996) 137-155.

[4] G. C. Hocking, "Cusp-like free-surface flows due to a submerged source or sink in the presence of a flat or sloping bottom", J. Austral. Math. Soc. Ser. B 26 (1985) 470-486.

[5] G. C. Hocking, "Critical withdrawal from a two-layer fluid through a line sink", J. Engng Maths 25 (1991) 1-11.

[6] G. C. Hocking, "Flow from a vertical slot into a layer of finite depth", Applied Math. Modelling 16 (1992) 300-306.

[7] G. C. Hocking, "Bow flows with smooth separation in water of finite depth", J. Austral. Math. Soc. Ser. $B 35$ (1993) 114-126.

[8] G. C. Hocking and J. M. Vanden-Broeck, "Withdrawal of a fluid of finite depth through a line sink with a cusp in the free surface", Computers and Fluids 27 (1998) 797-806.

[9] A. C. King and M. I. G. Bloor, "A note on the free surface induced by a submerged source at infinite Froude number", J. Austral. Math. Soc. Ser. B 30 (1988) 147-156.

[10] M. A. Madurasinghe, "Splashless ship bows with stagnant attachment", J. Ship Res. 32 (1988) 194-202.

[11] M. A. Madurasinghe and E. O. Tuck, "Ship bows with continuous and splashless flow attachment", J. Austral. Math. Soc. Ser. B 27 (1986) 442-452.

[12] S. W. McCue and L. K. Forbes, "Bow and stem flows with constant vorticity", J. Fluid Mech. 399 (1999) 277-300. 
[13] A. F. Teles da Silva and D. H. Peregrine, "Steep, steady surface waves on water of finite depth with constant vorticity", J. Fluid Mech. 195 (1988) 281-302.

[14] J. M. Vanden-Broeck, "Bow flows in water of finite depth", Phys. Fluids A 1 (1989) 1328-1330.

[15] J. M. Vanden-Broeck, "Steep solitary waves in water of finite depth with constant vorticity", $J$. Fluid Mech 274 (1994) 339-348.

[16] J. M. Vanden-Broeck, "Cusp flow due to a submerged source with a free surface partially covered by a lid", European J. Mech. B. (Fluids), 16 (1997) 249-255.

[17] J. M. Vanden-Broeck and J. B. Keller, "Free surface flow due to a sink", J. Fluid Mech. 175 (1987) $109-117$.

[18] J. M. Vanden-Broeck, L. W. Schwartz and E. O. Tuck, "Divergent low-Froude-number series expansion in nonlinear free-surface flow problems", Proc. R. Soc. Lond. A 361 (1978) 207-224.

[19] J. M. Vanden-Broeck and E. O. Tuck, "Computation of near-bow or stern flows, using series expansion in the Froude number", in Proc. 2nd Intl Conf. Numerical Ship Hydradynamics, Berkeley, CA (University Extension Publications, 1977), pp. 371-381. 